\title{
Understanding Rett Syndrome: By Means of Genetic Mutations and Evolutionary Classification
}

\author{
Dr. Ramneet Kaur ${ }^{1}$, Pratik Chatterjee ${ }^{2}$ \\ ${ }^{1}$ Assistant Professor, Department of Basic and Applied Sciences, RIMT University, Punjab, India \\ 1989ramneet[at]gmail.com
}

${ }^{2}$ School of BioSciences and Technology, M. Tech Biotechnology, Vellore Institute of Technology, Vellore - 632014 Tamil Nadu, India pratikchatterjee.mtech[at]gmail.com

\begin{abstract}
RETT (RTT) syndrome is a rare genetic disorder that becomes apparent in females at about 6-18 years of age accompanied with symptoms like seizures, sleeping problems, scoliosis, difficulty in walking, breathing etc. the affected females usually have a smaller head, a characteristic of the disease which is also characterized by repeated movement of the hand to the mouth known as mouthing. The disease occurs almost exclusively in males since it is a X-linked dominant disease and is lethal in males. MECP2(methyl CGP binding protein 2) gene codes for the MECP2 protein that is involved with the normal functioning of nerve cells, the protein binds to methylated DNA and then binds to other proteins to turn the gene off. Rett syndrome therefore more actively affects females, who, owing to X chromosome inactivation, have a mixture of cells that express either the wild-type or mutant version of MeCP2. The study has been discovered that alterations in cyclin-dependent kinase-like 5 (CDKL5) or forkhead box protein G1 (FOXG1) related to distinct neurodevelopmental disorders, caused by alterations in either one of these genes. The positions of three particular RTT-causing missense mutations-R133C, T158M and R306C, reflecting the spectrum of clinical severity among the affected patients. In this review study our aim is to provide insight to the structural features and properties, phylogenetic and interaction perspective of those mentioned proteins. This paper also indicates the disordered structure properties and evolution of those protein may provide worthy data for the development of therapeutic strategies of RTT
\end{abstract}

Keywords: Rett syndrome; cyclin-dependent kinase-like 5; forkhead box protein G1; methyl-CpG-binding protein 2; phylogenetic profile analysis; post-transcriptional modification ; therapeutic strategies

\section{Introduction}

Rett condition (RTT) is an atypical problem that was first represented by Andreas Rett in 1966 [1]. It is portrayed by serious debilitation, for example, deceleration of head development, loss of discourse, seizures, ataxia, development issue, and breathing aggravation [2]. Modifications in methyl CpG-restricting protein (MECP)2, a $\mathrm{X}$-connected quality engaged with the guideline of RNA grafting and chromatin rebuilding, were affirmed in roughly $95 \%$ of people determined to have RTT [3], while the others were affirmed in either cyclin-subordinate kinase-like (CDKL) 5 or fork head box protein (FOXG) 1 changes as atypical instances of RTT [4,5]. The transformations in MECP2 are commonly in a fatherly way inferred. Consequently, this disorder mostly influences young ladies, and the period of beginning shifts from 6 to year and a half $[2,6]$. Furthermore, Rett disorder can likewise influence guys with serious aggregate and early lethality following the inactivation of the sole X-connected duplicate of MECP2 [7]. In an uncommon case, it can likewise exist as physical mosaicism or co-happen with Klinefelter disorder in guys [8, 9]. Despite the fact that the causative qualities have been resolved, the inconsistent clinical aggregates respect the trouble in finding. Further, analysis might be trying the same number of the clinical highlights cover with those of other neurological and neurodevelopmental issues, and transformation in MECP2, FOXG1, and CDKL5 can likewise cause neurodevelopmental messes particular from RTT [10]. Accordingly, ensuing examinations have recommended that changes in either CDKL5 or FOXG1 should be delegated a particular problem from RTT as most of cases indicated a few contrasts in clinical highlights [11,
12, 13] Moreover, ongoing investigations have proposed that RTT is a monogenic issue brought about by transformations that adjust the usefulness of the methyl-CpG-restricting area (MBD) and the NCoR/SMRT cooperation space (NID) in MECP2 $[14,15,16]$. This may disentangle the complexity of building up a treatment technique. Be that as it may, explanation on the covered manifestations between those three proteins exhaustively on the sub-atomic premise additionally appears to be essential as the examination about it stays scant and it might give significant knowledge, especially for RTT.

The MeCP2 structure has been resolved utilizing different trial techniques, while the structure of FOXG1 has just been researched by expectations $[17,18]$. On account of CDKL5, the structure of the amino-terminal kinase space has just been distinguished, yet that of the long carboxy-terminal tail has not been explained [19]. Reestablishing Mecp2 quality capacity in a creature model abrogated the side effects of RTT. Development factor incitement (e.g., insulin-like development factor 1) and the initiation of synapse pathways (e.g., $\quad \beta 2$-adrenergic receptor pathway) can likewise incompletely save aggregates of Mecp2 knockout mice (RTT model mice), proposing that the issue is treatable $[20,21]$. Notwithstanding quality treatment, reactivation of an inactivated $\mathrm{X}$ chromosome is known to be another remedial strategy $[22,23]$. The restorative systems of RTT are a work in progress, and explanation on this perplexing problem needs different perspectives to make propels in arrangement. Despite the fact that RTT has been resolved as a monogenic issue, the complex organic framework propels us to essentially expand our viewpoint; also, MeCP2 contains a broad measure of confused districts which may 
encourage official with different accomplices. Considering a few focuses above, we explored the development and atomic highlights of MeCP2, CDKL5, and FOXG1 and their coupling accomplices utilizing phylogenetic profiling to increase a superior comprehension of their similitudes. Also, we anticipated the basic request problem affinity and evaluated the developmental rates per site of $\mathrm{MeCP} 2$, CDKL5, and FOXG1 to explore the connections between confused structure and other related properties with RTT.

\section{Rett Syndrome: Clinical Features and Stages}

The clinical conclusion of RTT depends on a battery of existing together and all around characterized comprehensive and restrictive standards [24, 25]. Following a time of typical neurological and actual improvement during the initial 6-year and a half of life, the main highlights of RTT start to show in youth and show up logically more than a few phases: stagnation (age 6-year and a half), fast relapse (age 1-4 years), pseudo stationary (age 2-conceivably life) and late engine weakening (age 10-life). Trademark side effects of RTT incorporate loss of obtained discourse and engine aptitudes, monotonous hand developments, breathing anomalies and seizures. RTT patients may likewise experience the ill effects of inconsistent scenes of gastrointestinal issues, hypoplasia, beginning stage osteoporosis, bruxism and shouting spells [24]. Regardless of these weaknesses, RTT patients are very much incorporated into their families and appreciate individual contact [26]. The advancement of new augmentative correspondence advances has permitted in any case non-verbal RTT patients to draw in with others and express themselves. Many youngsters determined to have RTT have diminished mind volume contrasted and sound people, predictable with a more modest head circuit $[27,28]$. Diminished mind volume is to a great extent because of little neuronal body size and a denser pressing of cells, especially in layers III and V of the cerebral cortex, thalamus, substantianigra, basal ganglia, amygdala, cerebellum and hippocampus [29]. Patients additionally have decreased dendritic arborization, demonstrative of a postponement in neuronal development [30]. Moreover, hypopigmentation of the substantianigra recommends a brokenness of dopaminergic neurons [31]. RTT patients show proof of dysregulated synapses, neuromodulators and carriers, demonstrating a significant part in synaptic capacity [32, 33].

Metabolic intricacies are additionally basic in RTT. Various patients present with dyslipidaemia [34, 35], raised plasma leptin and adiponectin [36, 37], raised alkali and aggravation of the gallbladder, an organ which stores bile for fat processing [38]. Changes in cerebrum starch digestion and neurometabolites related with cell respectability and layer turnover [39] have additionally been accounted for. Moreover, energy-creating mitochondria have unusual structure in patient cells. Reliably, modified electron transport chain complex capacity, expanded oxidative pressure, and raised degrees of lactate and pyruvate in blood and cerebrospinal liquid have been seen in RTT patients [40].
Treatment for RTT patients is presently restricted to indication control. With sufficient thoughtfulness regarding muscular intricacies, seizure control and nourishment, ladies with RTT may get by into middle age and more established. Notwithstanding, patients have an abrupt and startling demise pace of $26 \%$, a lot higher than solid people of a comparable age, and ordinarily bite the dust because of respiratory disease, heart precariousness and respiratory disappointment

\section{Materials \& Methods}

\subsection{Sequence Retrieval, Alignment, and Phylogenetic Analysis of MeCP2, CDKL5, and FOXG1 Proteins}

Orthologous groupings of human RTT and RTT-like causing proteins (MeCP2, CDKL5, and FOXG1) in chordates were recovered from the Kyoto Encyclopedia of Genes and Genomes (KEGG) succession comparability information base (https://www.kegg.jp/kegg/ssdb/) with a SmithWaterman likeness score limit of 100 and the bidirectional best hits (best-best hits) alternative. The most noteworthy likeness score for every species was utilized for every one of those proteins to limit repetition. Datasets were made for every protein and afterward adjusted utilizing MAFFT v.7 (https://mafft.cbrc.jp/arrangement/programming/) with the iterative refinement technique (FFT-NS-I), with a limit of 1000 cycles. Phylogenetic trees were built with the greatest probability strategy utilizing. The outgroup for each tree was chosen dependent on the NCBI Taxonomy Common Tree for the regular progenitor inside the dataset. Recreation of phylogenetic trees and estimation of models were acted in CIPRES Science Gateway.

\subsection{Structural Order-Disorder Prediction and Secondary Structure Predictions}

The underlying request problem inclination of every protein was anticipated utilizing IUPred2A (https://iupred2a.elte.hu/) utilizing the alternative for since quite a while ago disarranged areas. This forecast had values going from 0 (in number inclination for an arranged structure) to 1 (in number penchant for a cluttered structure), with 0.5 as the cut-off between the affinity for request and confusion. The outcomes for each site of every protein were planned onto its arrangement and taxon position in the phylogenetic tree utilizing iTOL(https://itol.embl.de/).

\subsection{Rate of Evolution per Site}

We determined the pace of advancement per site of human CDKL5, FOXG1, and MeCP2 comparative with their orthologs utilizing Rate4site (https://m.tau.ac.il/ itaymay/cp/rate4site.html). The adjusted successions of every protein dataset were determined utilizing the experimental Bayesian rule with the JTT model and 16 discrete classifications of the earlier gamma appropriation. Holes were treated as missing information, and yields were normalized as $\mathrm{Z}$ scores. The aftereffects of the pace of development of every buildup were then coordinated with the underlying request issue expectation result, and the circulation of the pace of advancement in the 
primary request and confusion of every protein was assessed with the Mann-Whitney U-test utilizing R programming.

\subsection{Point Mutations in MeCP2, CDKL5, and FOXG1}

Point changes in CDKL5, FOXG1, and MeCP2 were recognized from RettBASE (http://mecp2.chw.edu.au/). The measure of changes variations by and large in RettBASE are 929, 298, and 44 for MeCP2, CDKL5, and FOXG1, individually. We just chose missense changes that were related with pathogenic RTT. Also, non-pathogenic polymorphisms in everybody for examination were separated from the Exome Aggregation Consortium information base (http://exac.broadinstitute.org)

\subsection{Phylogenetic Profiling and Cluster Analyses of Human MeCP2, CDKL5, and FOXG1 and Their Interacting Proteins}

Arrangements of human MeCP2, CDKL5, and FOXG1 and their connection accomplices related to BioGRID (https://thebiogrid.org/) were acquired from the UniProtKB/Swiss-Prot information base (https://www.uniprot.org/help/uniprotkb) and utilized as the dataset. We created phylogenetic profiles of 326 eukaryotes in the KEGG information base (https://www.genome.jp/kegg/) utilizing the dataset as a question. Phylogenetic profiling is a technique for distinguishing the presence or nonappearance of orthologous proteins in an objective creature. The presence or nonappearance of proteins homologous to the question in every species was resolved utilizing KEGG Ortholog Cluster (https://www.genome.jp/devices/oc/), this instrument utilizes Smith-Waterman comparability scores of $\geq 150$ and symmetric likeness measures to arrange the ortholog qualities.

\section{Results}

\subsection{Atypical Rett syndrome variants.}

Table 1: These variants may be milder or more severe than classical RTT symptoms

\begin{tabular}{|c|c|}
\hline Type & Description \\
\hline $\begin{array}{c}\text { early-onset } \\
\text { seizure type }\end{array}$ & $\begin{array}{c}\text { seizures in the primary long periods of life by } \\
\text { indicating RTT syndrome, mutation in X-linked } \\
\text { cyclin-dependent kinase-like five gene }\end{array}$ \\
\hline $\begin{array}{c}\text { congenital } \\
\text { variant }\end{array}$ & $\begin{array}{c}\text { mutation in the Forkhead box G1( chromosome } \\
\text { 14), congenital microcephaly and intellectual } \\
\text { disability }\end{array}$ \\
\hline $\begin{array}{c}\text { late regression } \\
\text { type }\end{array}$ & RTT symptoms generated \\
\hline $\begin{array}{c}\text { 'Formefruste' } \\
\text { variant }\end{array}$ & $\begin{array}{c}\text { Atypical variant accounting for } 80 \% \text { of cases, } \\
\text { subtle neurological abnormalities }\end{array}$ \\
\hline
\end{tabular}

RTT patients are heterozygous for MECP2 change, conveying one typical and one transformed duplicate of MECP2. At the point when a youngster gives RTT-like indications, yet doesn't satisfy all the analytic measures for RTT, they might be determined to have atypical RTT, side effects of which veer off in time of beginning, succession of clinical profile as well as seriousness (table 1). Numerous atypical cases are related with changes in X-connected cyclin-subordinate kinase-like 5 (CDKL5) or Forkhead box
G1 (FOXG1), yet some stay unclear. Changes in MECP2 have likewise been related with scholarly inability, chemical imbalance and lupus erythematosis [41]

\subsection{Structural Order-Disorder Properties of RTT and RTT-like Causing Proteins during Chordate Evolution}

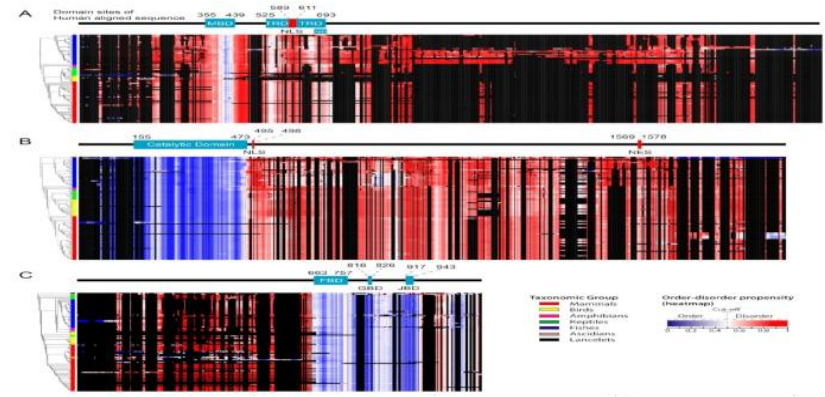

Figure 1: (A-C) Heat maps for MeCP2 (A), CDKL5 (B), and FOXG1 (C) are shown. MBD, TRD, NID, FBD, GBD, JBD, NLS, and NES indicate methyl-CpG-binding domain, transcriptional repression domain, NCoR/SMRT interaction

domain, forkhead binding domain, Groucho-binding

domain, JARID1B binding domain, nuclear localization signal, and nuclear export signal, respectively

Groupings of MeCP2, CDKL5, and FOXG1, individually, and built a warmth guide of the primary request issue affinity for every protein of these qualities as per adjusted successions and ordered situation in the phylogenetic tree (Figure 1)This examination was led to explore the transformative examples of underlying properties. The outcomes indicated that all proteins held both arranged and cluttered areas; by contrasting their dissemination with space and non-space districts, we found that the synergist area and non-area locales of CDKL5 were requested and disarranged, separately (Figure 1B). While most districts of MeCP2 were anticipated to be confused, some arranged structures were seen in the MBD. Moreover, FOXG1 indicated a shifted dissemination of requested scattered locales relating to area and non-space areas, with the previous anticipated to be completely requested (Figure $1 \mathrm{C}$ ). In spite of the fact that inclusions and cancellations were much of the time identified in scattered areas, especially in MeCP2 and FOXG1 (Figure $1 \mathrm{~A}, \mathrm{C})$, the underlying request problem of all proteins demonstrated to be steady in chordates, barring a couple conformational changes of FOXG1 and CDKL5 in warm blooded animals and fishes, individually. This demonstrated that the confused areas of MeCP2, CDKL5, and FOXG1 will in general be practical either as an entropic chain, transient restricting site, or lasting restricting site in chordates. Moreover, additions and cancellations were oftentimes distinguished in cluttered areas. This is brought about by their adaptability, which makes arrangement troublesome; a propensity of straight themes to lie among the adaptable cluttered areas; and the change of useful modules as for others during development that is conceivable in scattered districts.

\subsection{Phylogenetic Profiling of RTT and RTT-like Causing Proteins and Their Interaction Partners}

The examination recovered 240 human proteins cooperating with MeCP2, CDKL5, and FOXG1 from BioGRID and

\section{Volume 10 Issue 1, January 2021




\section{International Journal of Science and Research (IJSR)}

ISSN: 2319-7064

SJIF (2019): 7.583

UniProt information bases (Supplementary Table S5) $[44,45]$. To enlighten the interconnection of MeCP2, CDKL5, and FOXG1 restricting accomplices just as their developmental relationship, we led phylogenetic profiling and bunch examination of 326 eukaryotes utilizing the recovered groupings and the successions of the three proteins, MeCP2, CDKL5, and FOXG1, as inquiries (Figure 3, Supplementary Table S6). The outcomes indicated that the dataset was separated into four groups, which were characterized as Classes 1 to 4 . There were 58 saved proteins in chordates of Class 1, 92 in metazoans of Class 2, 17 in multicellular of Class 3, and 73 in eukaryotes of Class 4 . MeCP2 and CDKL5 had a place with Class 1, while FOXG1 had a place with Class 2 (Figure 2). FOXG1 and MECP2 appeared to have many restricting accomplices that go about as a record factor or quality articulation controller. Interestingly, CDKL5 will in general tie to a less number of proteins having capacities in directing cell bond, ciliogenesis, and cell multiplication; in any case, this protein has been appeared to collaborate with MeCP2. As RTT has been resolved to happen from the modified usefulness of MBD and NID of MECP2, we zeroed in on the broadly known restricting accomplices of these areas, for example, SIN3 record controller relative A (SIN3A), histone deacetylase (HDAC)1, and atomic receptor corepressor (NCOR) which assume parts as co-repressor edifices. Despite the fact that FOXG1 doesn't straightforwardly tie to MeCP2, we found that the coupling accomplices of MeCP2 co-repressor complex are additionally connected with FOXG1 restricting accomplices that likewise go about as co-repressor edifices, for example, extraordinary AT-rich arrangement restricting protein (SATB)2, lysine-explicit histone demethylase (KDM)1A, SWI/SNF-related network related actinsubordinate controller of chromatin subfamily (SMARC)A part 5, A-kinase anchor protein (AKAP)8, of which are antiquated proteins inside Classes 3 and 4 .

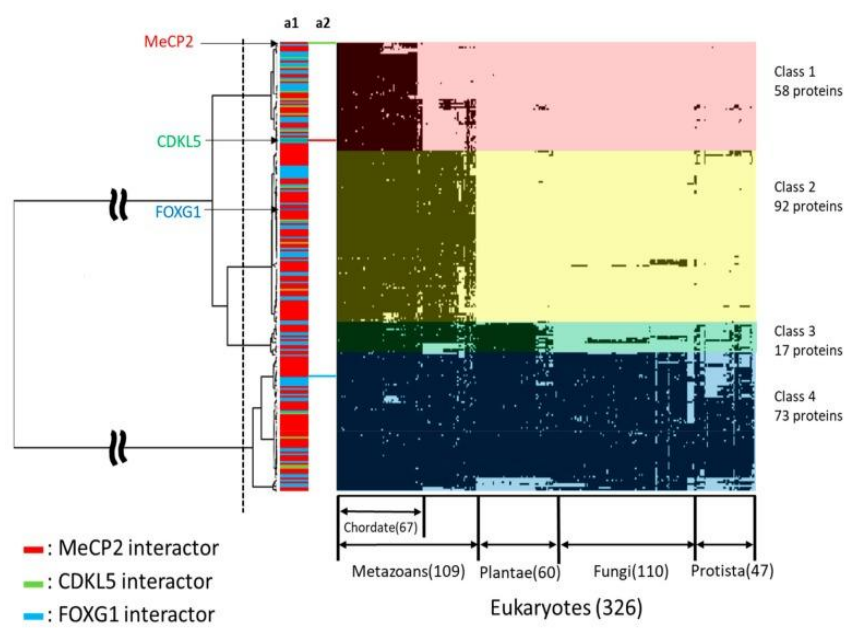

Figure 2: Phylogenetic profiling of MeCP2, CDKL5, and

FOXG1 proteins and their interaction partners. The

horizontal axis shows 326 eukaryotes for which whole genome sequences are available, and the vertical axis shows 240 human proteins related to RTT. Bar in a1 and a2 shows MeCP2-interactor (red), CDKL5-interactor (green),

FOXG1-interactor (blue), respectively.
4.4. Disease-Associated Missense Mutation Distribution in the Sequence of RTT and RTT-like Causing Proteins

Plotting missense transformations related with sicknesses may yield essential data on structure-work connections and the highlights of the protein. We explored missense changes in human MeCP2, CDKL5, and FOXG1 that were recently connected with pathogenic RTT from RettBASE and inspected the highlights of the related arrangements. There were 7,12 , and 18 individual amino corrosive locales in FOXG1, CDKL5, and MeCP2, separately, that held pathogenic missense transformations related or recently recommended to be related with pathogenic RTT (Figure 3). At the point when the frequencies were joined with those of cases noticed for every transformation, MeCP2 had a higher number of cases (1225) than CDKL5 (30) and FOXG1. Pathogenic RTT or RTT-like-related missense transformations were all the more habitually distinguished in area locales for all proteins, and in arranged and gradually advancing districts for MeCP2 and CDKL5 . Then again, numerous change destinations in $\mathrm{MeCP} 2$ were found near (or on account of Ser346Arg and Ser134Cys, covered with) phosphorylation locales (Figure 3), in spite of the fact that the recurrence of cases holding these transformation destinations was low (just one for each).

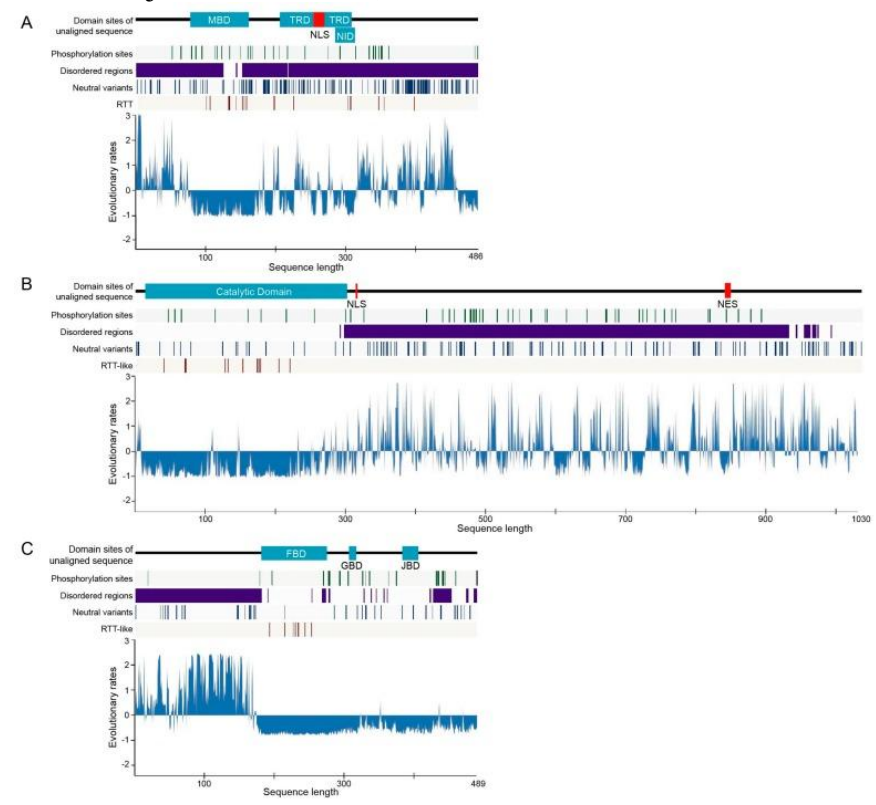

Figure 3: Pace of advancement per site in human RTTrelated proteins. (A-C) Rates of amino corrosive replacement in MeCP2 (A), CDKL5 (B), and FOXG1 (C) are appeared as blue zones. The bars above outlines demonstrate the situation of the space in the human grouping, with light blue territories showing the area and dark lines demonstrating no space. Preserved

phosphorylation destinations, confused district, single nucleotide polymorphisms in everyone, and pathogenic missense point change are plotted in green, purple, blue, and red lines, individually. The $\mathrm{x}$ and $\mathrm{y}$ tomahawks speak to the succession length and $\mathrm{Z}$ score of the transformative rates, separately.

\section{Discussion}

In neurons, MeCP2 fills in as extension between the NCoR1/SMRT-HDAC3 complex and DNA to encourage

Volume 10 Issue 1, January 2021 
transcriptional suppression, and transformations that influence MeCP2 official to the intricate reason RTT. While both Ncor1 and Hdac3 invalid mice pass on as incipient organisms, a neuron-explicit cancellation of Hdac3 produces feasible mice that continuously build up an aggregate fundamentally the same as that of Mecp2 invalid mice including irregular engine coordination, decreased friendliness and psychological deformities [42]. These information recommend that at any rate a subset of RTT-like aggregates in Mecp2 freak mice are brought about by the deficiency of HDAC3 in neurons. In addition, the function of the NCoR1/SMRT-HDAC3 in controlling lipogenesis in the Mecp2 mouse liver brings up fascinating issues about the part of this complex in directing lipids in the cerebrum. Natural chemistry considers have generally neglected to discover conclusive quality administrative focuses, to some extent, on the grounds that MeCP2 ties DNA at levels matching histone octamers [43]. Also, the cerebrum is a perplexing organ with a wide range of cell types in different phases of action, making entire mind examines hard to decipher. All things considered, at specific occasions and in explicit cells, MeCP2 is needed to secure the NCoR1/SMRT-HDAC3 complex to DNA, managing lipid creation in certain cells in the cerebrum as in the liver.

Metabolic dysregulation in RTT and different infections may grant considerable outcomes on downstream frameworks. At the point when one arm of digestion is bothered, it influences each interfacing pathway, including glucose, lipid, amino corrosive, nitrogen and mitochondrial respiratory digestion. Of course, both RTT patients and Mecp2 freak mice have strange reactions to glucose resilience tests, and freak mice are likewise insulin-safe [44]. Since Mecp2 mice specially utilize fat as opposed to glucose as their essential fuel source, almost certainly, other energy-detecting frameworks are influenced. In particular, energy digestion influences the post-translational alteration of proteins with $\mathrm{O}$-connected $\mathrm{N}$-acetylglucosamine (OGlcNAc), a supplement driven epigenetic controller [45, 46]. The guideline of O-GlcNAc is needed for some, proteins associated with neurogenesis, and bother of $\mathrm{O}$ GlcNAc protein alteration has just been related with numerous neurological sicknesses, including Alzheimer's $[45,46]$. Irregular cerebrum glucose-lipid homeostasis is likewise connected with oxidative pressure in Alzheimer's [44]. RTT patients have expanded oxidative weight and irregular mitochondrial structure, while RTT creature models have abandons in the mitochondrial respiratory chain and oxidative changes in the cerebrum [47]. Moreover, unusual lipid digestion is straightforwardly connected to aggravation [48], which is a part of pathology in both normal and atypical instances of RTT [48]. Quite, MeCP2 straightforwardly subdues Irak1 and down regulation of the NF- $\kappa \beta$ pathway improves side effects in Mecp2 invalid mice.

Over the most recent twenty years, exertion on explaining RTT has demonstrated a promising pattern towards building up a dependable treatment for this problem. Given a likeness in IDR properties and a few covering side effects, we examined the development of MeCP2, CDKL5, and FOXG1 scattered structures and their coupling accomplices through expectation and phylogenetic profiling, individually. Here, we gave understanding to the underlying attributes, advancement and collaboration scenes of those three proteins identified with RTT. We proposed that the scattered structures of MECP2, CDKL5, and FOXG1 add to the adaptability in mental health and may assume a critical part in mind development in chordates. We theoretically recommended that CDKL5 could be a likely objective for RTT treatment, especially by focusing on its confused structure that ranges after the reactant area to the $\mathrm{C}$-end, which shows plentiful straight themes that can tie to atoms with various structures of comparative liking. At last, this investigation may give important direction to test research, especially on the connection among RTT and scattered locales.

\section{Conclusion}

The comprehension of metabolic parts of RTT pathology uncovers likely remedial mediations. Statin drugs represent just a single group of various metabolic modulators being created to treat lipid collection or insulin obstruction in Type II diabetes, which might be repurposed to treat RTT. Consistently, new medication medicines are tried in Mecp2 creature models that salvage various parts of RTT aggregates. For instance, the treatment of Mecp2 invalid mice with a protein-tyrosine phosphatase 1B (PTP1B) rival that was created to treat Type II diabetes broadened life expectancy, diminished hindlimb catching and improved engine execution [49]. Also, Trolox, a nutrient E subsidiary, standardized blood glucose levels, diminished oxidative pressure and improved exploratory conduct in Mecp2 mice [50]. Moreover, insulin-like development factor (IGF-1) incompletely saved locomotor action, respiratory capacity and pulse in Mecp2 mice [209,210]. Notwithstanding statins, stage 2 clinical preliminaries for RTT are in advancement for a rundown of potential treatments including IGF-1 (NCT01777542), EPI-743 (NCT01822249), triheptanoin (NCT03059160) and NNZ-2566 (NCT01703533). In any case, in spite of this advancement, no single treatment has yet fixed each aggregate in Mecp2 mice or yet demonstrated to successfully treat RTT side effects. Moreover, changes in individuals from the NCoR1/SMRT-HDAC3 complex should be inspected for functions in other youth neurological sicknesses. As of now, changes in different segments of the complex, TBLR1 and TBL1, have been related with chemical imbalance, scholarly incapacity, Pierpont condition, an issue portrayed by formative postponement and unusual fat dissemination in the distal appendages, and West disorder, a problem with RTTlike highlights.

\section{Acknowledgement}

I express my gratitude to everyone who supported me and to Dr. Ramneet Kaur, for the keen interest she took in me. She explained to me solutions to the problems that had a straining effect. Without her help it was a matter of acute impossibility to propel in this endeavor. It is a matter of profound privilege, and pleasure to pay my sincere and heartfelt thanks to her. I am also very grateful to editorial staff who edited the manuscript.

\section{Volume 10 Issue 1, January 2021 www.ijsr.net}




\section{References}

[1] Rett A. On a unusual brain atrophy syndrome in hyperammonemia in childhood. Wien. Med. Wochenschr. 1966;116:723-726

[2] Hanefeld F. The clinical pattern of the Rett syndrome. Brain Dev. 1985;7:320-325. doi: 10.1016/S03877604(85)80037-1.

[3] Laurvick C.L., De Klerk N., Bower C., Christodoulou J., Ravine D., Ellaway C., Williamson S., Leonard H. Rett syndrome in Australia: A review of the epidemiology. J. Pediatr. 2006;148:347-352. doi: 10.1016/j.jpeds.2005.10.037

[4] Ariani F., Hayek G., Rondinella D., Artuso R., Mencarelli M.A., Spanhol-Rosseto A., Pollazzon M., Buoni S., Spiga O., Ricciardi S., et al. FOXG1 is responsible for the congenital variant of Rett syndrome. Am. J. Hum. Genet. 2008;83:89-93. doi: 10.1016/j.ajhg.2008.05.015

[5] Weaving L.S., Christodoulou J., Williamson S.L., Friend K.L., McKenzie O.L., Archer H., Evans J., Clarke A., Pelka G.J., Tam P.P., et al. Mutations of CDKL5 cause a severe neurodevelopmental disorder with infantile spasms and mental retardation. Am. J. Hum. Genet. 2004;75:1079-1093. doi: 10.1086/426462

[6] Trappe R., Laccone F., Cobilanschi J., Meins M., Huppke P., Hanefeld F., Engel W. MECP2. mutations in sporadic cases of Rett syndrome are almost exclusively of paternal origin. Am. J. Hum. Genet. 2001;68:1093-1101. doi: 10.1086/320109

[7] Van Esch H., Bauters M., Ignatius J., Jansen M., Raynaud M., Hollanders K., Lugtenberg D., Bienvenu T., Jensen L.R., Gecz J., et al. Duplication of the MECP2 region is a frequent cause of severe mental retardation and progressive neurological symptoms in males. Am. J. Hum. Genet. 2005;77:442-453. doi: $10.1086 / 444549$.

[8] Clayton-Smith J., Watson P., Ramsden S., Black G.C.M. Somatic mutation in MECP2 as a non-fatal neurodevelopmental disorder in males. Lancet. 2000;356:830-832. doi: 10.1016/S01406736(00)02661-1.

[9] Ben Zeev B., Yaron Y., Schanen N.C., Wolf H., Brandt N., Ginot N., Shomrat R., Orr-Urtreger A. Rett syndrome: Clinical manifestations in males with MECP2 mutations. J. Child. Neurol. 2002;17:20-24. doi: $10.1177 / 088307380201700105$.

[10] Neul J.L. The relationship of Rett syndrome and MECP2 disorders to autism. Dialogues Clin. Neurosci. 2012;14:253-262.

[11] Fehr S., Wilson M., Downs J., Williams S., Murgia A., Sartori S., Vecchi M., Ho G., Polli R., Psoni S., et al. The CDKL5 disorder is an independent clinical entity associated with early-onset encephalopathy. Eur. J. Hum. Genet. 2013;21:266-273. doi: 10.1038/ejhg.2012.156.

[12] Hector R.D., Kalscheuer V.M., Hennig F., Leonard H., Downs J., Clarke A., Benke T.A., Armstrong J., Pineda M., Bailey M.E.S., et al. CDKL5 variants: Improving our understanding of a rare neurologic disorder. Neurol. Genet. 2017;3:e200. doi: 10.1212/NXG.0000000000000200.
[13] Kortum F., Das S., Flindt M., Morris-Rosendahl D.J., Stefanova I., Goldstein A., Horn D., Klopocki E., Kluger G., Martin P., et al. The core FOXG1 syndrome phenotype consists of postnatal microcephaly, severe mental retardation, absent language, dyskinesia, and corpus callosum hypogenesis. J. Med. Genet. 2011;48:396-406. doi: 10.1136/jmg.2010.087528.

[14] Lyst M.J., Ekiert R., Ebert D.H., Merusi C., Nowak J., Selfridge J., Guy J., Kastan N.R., Robinson N.D., de Lima Alves F., et al. Rett syndrome mutations abolish the interaction of MeCP2 with the NCoR/SMRT corepressor. Nat. Neurosci. 2013;16:898-902. doi: 10.1038/nn.3434

[15] Lyst M.J., Bird A. Rett syndrome: A complex disorder with simple roots. Nat. Rev. Genet. 2015;16:261-275. doi: $10.1038 / \mathrm{nrg} 3897$.

[16] Tillotson R., Selfridge J., Koerner M.V., Gadalla K.K.E., Guy J., De Sousa D., Hector R.D., Cobb S.R., Bird A. Radically truncated $\mathrm{MeCP} 2$ rescues Rett syndrome-like neurological defects. Nature. 2017;550:398-401. doi: 10.1038/nature24058.

[17] Ghosh R.P., Nikitina T., Horowitz-Scherer R.A., Gierasch L.M., Uversky V.N., Hite K., Hansen J.C., Woodcock C.L. Unique physical properties and interactions of the domains of methylated DNA binding protein 2. Biochemistry. 2010;49:4395-4410. doi: 10.1021/bi9019753.

[18] Toth-Petroczy A., Palmedo P., Ingraham J., Hopf T.A., Berger B., Sander C., Marks D.S. Structured States of Disordered Proteins from Genomic Sequences. Cell. 2016;167:158-170. doi: 10.1016/j.cell.2016.09.010.

[19] Tropea D., Giacometti E., Wilson N.R., Beard C., McCurry C., Fu D.D., Flannery R., Jaenisch R., Sur M. Partial reversal of Rett Syndrome-like symptoms in MeCP2 mutant mice. Proc. Natl. Acad. Sci. USA. 2009;106:2029-2034. doi: 10.1073/pnas.0812394106

[20] Carrette L.L.G., Wang C.Y., Wei C., Press W., Ma W., Kelleher R.J., 3rd, Lee J.T. A mixed modality approach towards $\mathrm{Xi}$ reactivation for Rett syndrome and other X-linked disorders. Proc. Natl. Acad. Sci. USA. 2018;115:E668-E675. doi: 10.1073/pnas.1715124115

[21] Shah R.R., Bird A.P. MeCP2 mutations: Progress towards understanding and treating Rett syndrome. Genome Med. 2017;9:17. doi: 10.1186/s13073-0170411-7.

[22] Mauri F., McNamee L.M., Lunardi A., Chiacchiera F., Del Sal G., Brodsky M.H., Collavin L. Modification of Drosophila p53 by SUMO modulates its transactivation and pro-apoptotic functions. J. Biol. Chem. 2008;283:20848-20856. doi: 10.1074/jbc.M710186200

[23] Dyson H.J., Wright P.E. Intrinsically unstructured proteins and their functions. Nat. Rev. Mol. Cell. Biol. 2005;6:197-208. doi: 10.1038/nrm1589

[24] Hagberg B. 2002. Clinical manifestations and stages of Rett syndrome. Ment. Retard. Dev. Disabil. Res. Rev. 8, 61-65.

[25] Hagberg B, Witt-Engerström I, Opitz JM, Reynolds JF. 1986. Rett syndrome: a suggested staging system for describing impairment profile with increasing age towards adolescence. Am. J. Med. Genet. 25, 47-59.

[26] Neul JL, et al. 2010. Rett syndrome: revised diagnostic 
criteria and nomenclature. Ann. Neurol. 68, 944-950. (doi:10.1002/ana.22124)

[27] Haas RH. 1988. The history and challenge of Rett syndrome. J. Child Neurol. 3, S3-S5.

[28] Townend GS, Marschik PB, Smeets E, van de Berg R, van den Berg M, Curfs LMG. 2016. Eye gaze technology as a form of augmentative and alternative communication for individuals with Rett syndrome: experiences of families in the Netherlands. J. Dev. Phys. Disabil. 28, 101-112. (doi:10.1007/s10882-0159455-z)

[29] Jellinger K, Seitelberger F, Opitz JM, Reynolds JF. 1986. Neuropathology of Rett syndrome. Am. J. Med. Genet. 25, 259-288. (doi:10.1002/ajmg.1320250528)

[30] Jellinger K, Armstrong D, Zoghbi HY, Percy AK. 1988. Neuropathology of Rett syndrome. Acta Neuropathol.. 76, 142-158. (doi:10.1007/BF00688098)

[31] Armstrong DD. 1992. The neuropathology of the Rett syndrome. Brain Dev. 14, S89-S98. [PubMed]

[32] Kaufmann WE, Moser HW. 2000. Dendritic anomalies in disorders associated with mental retardation. Cereb. Cortex 10, 981-991. (doi:10.1093/cercor/10.10.981)

[33] Taneja P, Ogier M, Brooks-Harris G, Schmid DA, Katz DM, Nelson SB. 2009. Pathophysiology of locus ceruleus neurons in a mouse model of Rett syndrome. J. Neurosci. 29, $12 \quad 187-12 \quad 195$. (doi:10.1523/JNEUROSCI.3156-09.2009)

[34] Zoghbi HY, Milstien S, Butler IJ, Smith EOB, Kaufman S, Glaze DG, Percy AK. 1989. Cerebrospinal fluid biogenic amines and biopterin in rett syndrome. Ann. Neurol. 25, 56-60. (doi:10.1002/ana.410250109)

[35] Justice MJ, Buchovecky CM, Kyle SM, Djukic A. 2013. A role for metabolism in Rett syndrome pathogenesis: new clinical findings and potential treatment targets. Rare Dis. 1, e27265 (doi:10.4161/rdis.27265)

[36] Segatto M, Trapani L, Di Tunno I, Sticozzi C, Valacchi G, Hayek J, Pallottini V. 2014. Cholesterol metabolism is altered in Rett syndrome: a study on plasma and primary cultured fibroblasts derived from patients. PLoS ONE 9, e104834 (doi:10.1371/journal.pone.0104834)

[37] Acampa M, Guideri F, Hayek J, Blardi P, De Lalla A, Zappella M, Auteri A. 2008. Sympathetic overactivity and plasma leptin levels in Rett syndrome. Neurosci. Lett. 432, 69-72. (doi:10.1016/j.neulet.2007.12.030)

[38] Blardi P, De Lalla A, D'Ambrogio T, Vonella G, Ceccatelli L, Auteri A, Hayek J. 2009. Long-term plasma levels of leptin and adiponectin in Rett syndrome. Clin. Endocrinol. 70, 706-709. (doi:10.1111/j.1365-2265.2008.03386.x)

[39] Clinical investigation, and management of gallbladder disease in Rett syndrome. Dev. Med. Child Neurol. 56, 756-762. (doi:10.1111/dmcn.12358)

[40] Matsuishi T, Urabe F, Percy AK, Komori H, Yamashita Y, Schultz RS, Ohtani Y, Kuriya N, Kato H. 1994. Abnormal carbohydrate metabolism in cerebrospinal fluid in Rett syndrome. J. Child Neurol. 9, 26-30.

[41] Functional characterization of the MECP2/IRAK1 lupus risk haplotype in human $\mathrm{T}$ cells and a human MECP2 transgenic mouse.Koelsch KA, Webb R,
Jeffries M, Dozmorov MG, Frank MB, Guthridge JM, James JA, Wren JD, Sawalha AHJ Autoimmun. 2013 Mar; 41():168-74.

[42] Histone deacetylase 3 associates with $\mathrm{MeCP} 2$ to regulate FOXO and social behavior.Nott A, Cheng J, Gao F, Lin YT, Gjoneska E, Ko T, Minhas P, Zamudio AV, Meng J, Zhang F, Jin P, Tsai LHNat Neurosci. 2016 Nov; 19(11):1497-1505.

[43] Neuronal MeCP2 is expressed at near histone-octamer levels and globally alters the chromatin state.Skene PJ, Illingworth RS, Webb S, Kerr AR, James KD, Turner DJ, Andrews R, Bird APMol Cell. 2010 Feb 26; 37(4):457-68.

[44] MeCP2 co-ordinates liver lipid metabolism with the NCoR1/HDAC3 corepressor complex.Kyle SM, Saha PK, Brown HM, Chan LC, Justice MJHum Mol Genet. 2016 Jul 15; 25(14):3029-3041

[45] Abnormalities of thyroid function and glucose control in subjects with Rett syndrome.Cooke DW, Naidu S, Plotnick L, Berkovitz GDHorm Res. 1995; 43(6):2738.

[46] Oxidative brain damage in Mecp2-mutant murine models of Rett syndrome.De Felice C, Della Ragione F, Signorini C, Leoncini S, Pecorelli A, Ciccoli L, Scalabrì F, Marracino F, Madonna M, Belmonte G, Ricceri L, De Filippis B, Laviola G, Valacchi G, Durand T, Galano JM, Oger C, Guy A, Bultel-Poncé V, Guy J, Filosa S, Hayek J, D'Esposito MNeurobiol Dis. 2014 Aug; 68():66-77.

[47] Liver $X$ receptors link lipid metabolism and inflammation.Schulman IGFEBS Lett. 2017 Oct; 591(19):2978-2991.

[48] Cytokine Dysregulation in MECP2- and CDKL5Related Rett Syndrome: Relationships with Aberrant Redox Homeostasis, Inflammation, and $\omega-3$ PUFAs.Leoncini S, De Felice C, Signorini C, Zollo G, Cortelazzo A, Durand T, Galano JM, Guerranti R, Rossi M, Ciccoli L, Hayek JOxid Med Cell Longev. 2015; 2015()$: 421624$.

[49] PTP1B inhibition suggests a therapeutic strategy for Rett syndrome.Krishnan N, Krishnan K, Connors CR, Choy MS, Page R, Peti W, Van Aelst L, Shea SD, Tonks NKJ Clin Invest. 2015 Aug 3; 125(8):3163-77.

[50] Systemic Radical Scavenger Treatment of a Mouse Model of Rett Syndrome: Merits and Limitations of the Vitamin E Derivative Trolox.Janc OA, Hüser MA, Dietrich K, Kempkes B, Menzfeld C, Hülsmann S, Müller MFront Cell Neurosci. 2016; 10():266. 